\title{
Study of Nitrogen Release Potential of Entisol and Vertisol as Influenced by Various Sources of Nitrogenous Fertilizers
}

\author{
N.B. More, S.S. Sadafule*, V.R. Gaikwad, B.D. Tamboli and G.D. Patil \\ Division of Soil Science and Agriculture Chemistry, College of Agriculture, Pune, \\ Maharashtra, India \\ *Corresponding author
}

\section{A B S T R A C T}

\section{Keywords \\ Nitrogen release potential, Entisol, Vertisol, Nitrogenous fertilizers}

Article Info

Accepted:

20 December 2018

Available Online:

10 January 2019
The experiment was conducted to study the nitrogen release potential of Entisol and Vertisolas influenced by the treatments of neem coated urea (NCU), DAP, NPK briquette, NP briquette, urea briquette and crotonylidene diurea (CDU). The application of all the sources of nitrogenous fertilizers increased the $\mathrm{N}$ release potential by $336.78 \%$ to $411.49 \%$ over the control in Entisol and by $342.59 \%$ to $470.98 \%$ over the control in Vertisol. The application of NCU in Entisol(7.16 mg $\mathrm{kg}^{-1}$ day $\left.^{-1}\right)$ and the application of NPK briquette in Vertisol $\left(7.63 \mathrm{mg} \mathrm{kg}^{-1}\right.$ day $\left.^{-1}\right)$ showed the highest $\mathrm{N}$ release potential. The NPK briquette and NP briquette are identified as the best slow nitrogen releasing fertilizers followed by the neem coated urea and urea briquette.

\section{Introduction}

The improved understanding of $\mathrm{N}$ mineralization and $\mathrm{N}$ immobilization, along with their continuous changing dynamics may improve our ability to manage $\mathrm{N}$ cycling and increase nitrogen use efficiency (NUE) by minimizing $\mathrm{N}$ losses whatever the form (Cabrera et al., 2005). The use of slow $\mathrm{N}$ releasing fertilizers having higher nitrogen release potential is new development in this direction of improving NUE of fertilizers. To minimize nutrient losses and increase the use efficiency of $\mathrm{N}$ fertilizer, the placement of fertilizer or spot application of fertilizer, use of slow release fertilizer and nitrification inhibitors are recommended. The use of urea super granules, urea briquette and urea DAP briquette are another development in this direction (Daftardar and Savant, 1995). Placement of NPK briquette at $10 \mathrm{~cm}$ deep maintained higher level of $\mathrm{NH}_{4}{ }^{+}-\mathrm{N}$ and $\mathrm{NO}_{3}{ }^{-}-$ $\mathrm{N}$ in soil (More, 1999). However, the information regarding a comparative performance of neem coated urea, DAP, NPK briquette, NP briquette, urea briquette and crotonylidene diurea with respect to nitrogen release pattern and availability of $\mathrm{NH}_{4}{ }^{+}-\mathrm{N}$ and $\mathrm{NO}_{3}{ }^{-} \mathrm{N}$ from these sources is limited. Therefore, the present study was undertaken 
to study the nitrogen release potential of Entisol and Vertisol as affected by these various sources of nitrogenous fertilizers.

\section{Materials and Methods}

The present laboratory experiment was conducted at Division of Soil Science and Agricultural Chemistry, College of Agriculture, Pune, Maharashtra during 201718 to study the release pattern of nitrogen in Entisol and Vertisol due to effect of various inorganic nitrogenous fertilizers at field capacity moisture regime $(0.33$ bar). The various physico-chemical properties of soils are analyzed by using various standard methods, the soil properties are given in given in table 1 .

There were fourteen treatments in experiment viz. combination of six nitrogenous fertilizers viz. $\mathrm{F}_{1}$-neem coated urea (NCU), $\mathrm{F}_{2}$-DAP, $\mathrm{F}_{3}$ NPK briquette, $\mathrm{F}_{4}$-NP briquette, $\mathrm{F}_{5}$-urea briquette and $\mathrm{F}_{6}$-Crotonylidene diurea (CDU) and $\mathrm{F}_{0}$-control with two soils viz. Entisol $\left(\mathrm{S}_{1}\right)$ and $\operatorname{Vertisol}\left(\mathrm{S}_{2}\right)$. For maintaining moisture at field capacity level, double distilled water was used throughout the experiment. The amount of $\mathrm{N}$ fertilizers to be added is calculated on the basis of recommended dose of rice crop i.e. $100 \mathrm{~kg} \mathrm{~N}$ per hectare. As 1 ha of soil weight is $2.24 \times 10^{6} \mathrm{~kg}$ so further calculations were made to determine the quantity of $\mathrm{N}$ fertilizers for $1 \mathrm{~kg}$ of soil and $200 \mathrm{mg}$ of $\mathrm{N}$ was added per $\mathrm{kg}$ of soil (Table 2).

Incubation study for $0,15,30,45,60,75$ and 90 days of incubation (DOI) after addition of nitrogenous fertilizers into soils was carried out at ambient condition. The $\mathrm{NH}_{4}{ }^{+}-\mathrm{N}$ and $\mathrm{NO}_{3}{ }^{-}-\mathrm{N}$ in Entisol and Vertisol are evaluated by method described by Kenney and Nelson (1982). It is determined immediately after sampling at each interval day by taking $5 \mathrm{gm}$ of soil from each incubated bowl. At the same time same weight of soil sample was kept for determining the moisture content for further calculations.

\section{Results and Discussion}

\section{Nitrogen release potential of Entisol}

Among the different nitrogenous fertilizers, the highest amount of cumulative $\mathrm{NH}_{4}{ }^{+}-\mathrm{N}$ $\left(153.03 \mathrm{mg} \mathrm{kg}^{-1}\right.$ ) found in DAP whereas the highest amount of $\mathrm{NO}_{3}{ }^{-} \mathrm{N}\left(596.59 \mathrm{mg} \mathrm{kg}^{-1}\right)$ found in NPK briquette fertilizer (Table 3). In case of $\mathrm{N}$ release potential, thehighest nitrogen release rate was observed in case of fertilizer $\mathrm{F}_{1}$ (NCU) @ $7.16 \mathrm{mg} \mathrm{kg}^{-1}$ day $^{-1}$ followed by fertilizer $\mathrm{F}_{3}$ (NPK briquette) @ $6.98 \mathrm{mg} \mathrm{kg}^{-1}$ day and fertilizer $\mathrm{F}_{4} @ 6.94 \mathrm{mg}$ $\mathrm{kg}^{-1} \mathrm{day}^{-1}$ in Entisol. Similarly Suganya et al., (2009) observed that the lowest nitrate nitrogen content was under NCU products which show the higher $\mathrm{N}$ release potential with respect to time. Thus, use of neem coated urea products prolonged the nitrogen availability for the crop growth thereby minimized the losses of nitrogen and improved the nitrogen use efficiency.

The lowest nitrogen release rate found to be in control fertilizer@ $1.74 \mathrm{mg} \mathrm{kg}^{-1} \mathrm{day}^{-1}$.It is observed that nitrogen release potential was highest in all fertilizer soils than control. Further it was also found that at the end of incubation period $\mathrm{NO}_{3}{ }^{-}-\mathrm{N}$ was at higher levels than $\mathrm{NH}_{4}{ }^{+}-\mathrm{N}$ in all treatments which might be due to activities of nitrifying microbes which oxidize the $\mathrm{NH}_{4}{ }^{+}-\mathrm{N}$ to $\mathrm{NO}_{3}{ }^{-}-\mathrm{N}$. Similar results were observed by Singh (2017) while studying mineralization kinetics of organic manures (Table 4 and 5).

During study the application of all the sources of nitrogenous fertilizers increased the $\mathrm{N}$ release potential by $336.78 \%$ to $411.49 \%$ over the control.The nitrogen release potential in Entisolwas in order; NCU >NPK briquette $>$ NP briquette $>$ DAP $>$ UB $>$ CDU $>$ Control . 


\section{Nitrogen release potential of Vertisol}

Among the different nitrogenous fertilizers, the highest amount of cumulative $\mathrm{NH}_{4}{ }^{+}-\mathrm{N}$ (154.10 $\mathrm{mg} \mathrm{kg}^{-1}$ ) found in DAP whereas the highest amount of $\mathrm{NO}_{3}-\mathrm{N}\left(643.83 \mathrm{mg} \mathrm{kg}^{-1}\right)$ found in NPK briquette fertilizer.

The highest quantity of nitrogen release potential was observed in NPK briquette @ $7.63 \mathrm{mg} \mathrm{kg}^{-1}$ day $^{-1}$ followed by NP briquette @ $7.57 \mathrm{mg} \mathrm{kg}^{-1}$ day $^{-1}$ and Urea briquette @ $7.31 \mathrm{mg} \mathrm{kg}^{-1}$ day throughout the incubation study. As that of Entisol, the quantity of $\mathrm{NO}_{3}$ $-\mathrm{N}$ found to be very high than that of $\mathrm{NH}_{4}{ }^{+}-\mathrm{N}$ at the end of incubation study.
The order of nitrogen release potential was found to be; NPK briquette $>\mathrm{NP}$ briquette $>\mathrm{UB}>\mathrm{DAP}>\mathrm{NCU}>\mathrm{CDU}>$ Control. Also in Vertisol, CDU showed the lowest nitrogen release potential as that of Entisol. From the results, it is revealed that neem coated urea $\left(\mathrm{F}_{1}\right)$ in Entisol while NPK briquette $\left(\mathrm{F}_{3}\right)$ in Vertisol performed better nitrogen release potential than other fertilizers in same soils. This proves the superiority of briquette fertilizers as reported by More and Shinde (2001), Durgude et al., (2008) and Singh (2012). The application of all the sources of nitrogenous fertilizers increased the $\mathrm{N}$ release potential by 342.59 per cent to 470.98 per cent over the control in Vertisol (Table 6-8).

Table.1 Physico-chemical properties of Entisol and Vertisol

\begin{tabular}{|c|c|c|c|}
\hline Sr. No. & Soil properties & Entisol & Vertisol \\
\hline A. & \multicolumn{3}{|l|}{ Physical properties } \\
\hline 1. & Sand $(\%)$ & 52.50 & 20.35 \\
\hline 2. & Silt (\%) & 31.75 & 28.05 \\
\hline 3. & Clay (\%) & 15.75 & 51.60 \\
\hline 4. & Textural class & Sandy loam & Clay \\
\hline 5. & Bulk density $\left(\mathrm{g} \mathrm{cm}^{-3}\right)$ & 1.45 & 1.27 \\
\hline 6. & Field capacity (\%) & 29.02 & 37.60 \\
\hline 7. & Permanent wilting point (\%) & 15.54 & 20.60 \\
\hline B. & \multicolumn{3}{|l|}{ Chemical properties } \\
\hline 8. & pH (1:2.5; soil:water) & 7.31 & 8.14 \\
\hline 9. & $\mathrm{EC}\left(\mathrm{dSm}^{-1}\right)$ & 0.12 & 0.23 \\
\hline 10. & Organic carbon $(\%)$ & 0.28 & 0.54 \\
\hline 11. & $\mathrm{CaCO}_{3}$ equivalent $(\%)$ & 1.75 & 8.01 \\
\hline 12. & Available nitrogen $\left(\mathrm{kg} \mathrm{ha}^{-1}\right)$ & 213.24 & 288.51 \\
\hline 13. & Available phosphorous $\left(\mathrm{kg} \mathrm{ha}^{-1}\right)$ & 34.50 & 24.38 \\
\hline 14. & Available potassium $\left(\mathrm{kg} \mathrm{ha}^{-1}\right)$ & 329.28 & 499.52 \\
\hline 15. & Ammonical nitrogen $\left(\mathrm{mg} \mathrm{kg}^{-1}\right)$ & 13.05 & 19.60 \\
\hline 16. & Nitrate nitrogen $\left(\mathrm{mg} \mathrm{kg}^{-1}\right)$ & 22.60 & 31.20 \\
\hline \multirow[t]{4}{*}{17.} & Exchangeable cations (meq. $100 \mathrm{~g}^{-1}$ ) & & \\
\hline & $\mathrm{Mg}^{2+}$ & $\begin{array}{l}20.29 \\
13.80\end{array}$ & $\begin{array}{l}01.30 \\
26.10\end{array}$ \\
\hline & $\mathrm{Na}^{+}$ & 21.35 & 29.84 \\
\hline & $\mathrm{k}^{+}$ & 23.40 & 21.09 \\
\hline
\end{tabular}


Table.2 Quantity of nitrogenous fertilizers used for incubation studies

\begin{tabular}{|l|c|c|}
\hline Sources of N fertilizers & $\begin{array}{c}\text { Estimated Total } \\
\text { N content }(\mathbf{\%})\end{array}$ & $\begin{array}{c}\text { Amount of N fertilizers (mg) added } \\
\text { to maintain 200 mg N kg } \mathbf{~ s o i l ~}\end{array}$ \\
\hline Neem coated urea & 43.05 & 193.80 \\
\hline DAP & 16.10 & 496.00 \\
\hline NPK briquette & 25.66 & 327.60 \\
\hline NP briquette & 32.66 & 256.00 \\
\hline Urea briquette & 42.00 & 193.80 \\
\hline Crotonylidene diurea & 32.50 & $226.00($ micro ml) \\
\hline
\end{tabular}

Table.3 Effect of nitrogenous fertilizers on cumulative $\mathrm{NH}_{4}{ }^{+}-\mathrm{N}$ content of Entisol $\left(\mathrm{mg} \mathrm{kg}^{-1}\right)$

\begin{tabular}{|l|c|c|c|c|c|c|c|c|}
\hline \multirow{2}{*}{$\begin{array}{c}\text { Nitrogenous } \\
\text { fertilizers }\end{array}$} & \multicolumn{7}{|c|}{ Incubation periods (Days) } & Cumulativ \\
\cline { 2 - 9 } & 0 & 15 & 30 & 45 & 60 & 75 & 90 & e total \\
\hline NCU & 30.80 & 26.73 & 25.50 & 11.19 & 7.94 & 7.30 & 5.15 & 114.60 \\
\hline DAP & 75.00 & 24.10 & 22.73 & 11.55 & 8.31 & 7.22 & 4.12 & 153.03 \\
\hline $\begin{array}{l}\text { NPK } \\
\text { briquette }\end{array}$ & 31.03 & 20.80 & 11.80 & 10.97 & 8.40 & 8.67 & 7.30 & 98.97 \\
\hline NP briquette & 33.83 & 24.30 & 12.15 & 11.35 & 9.48 & 8.62 & 7.03 & 106.76 \\
\hline UB & 29.13 & 25.20 & 13.03 & 9.59 & 7.04 & 7.18 & 3.99 & 95.16 \\
\hline CDU & 44.87 & 25.50 & 20.74 & 12.16 & 6.14 & 6.33 & 5.29 & 121.04 \\
\hline Control & 14.97 & 13.30 & 12.98 & 8.59 & 7.23 & 6.95 & 3.20 & 67.22 \\
\hline
\end{tabular}

Table.4 Effect of nitrogenous fertilizers on cumulative $\mathrm{NO}_{3}{ }^{-} \mathrm{N}$ content of Entisol $\left(\mathrm{mg} \mathrm{kg}^{-1}\right)$

\begin{tabular}{|l|c|c|c|c|c|c|c|c|}
\hline \multirow{2}{*}{$\begin{array}{c}\text { Nitrogenous } \\
\text { fertilizers }\end{array}$} & \multicolumn{7}{|c|}{ Incubation periods (Days) } & Cumulativ \\
\cline { 2 - 9 } & 0 & 15 & 30 & 45 & 60 & 75 & 90 & e total \\
\hline NCU & 14.57 & 39.51 & 85.80 & 86.26 & 97.42 & 125.45 & 126.0 & 575.01 \\
\hline DAP & 15.63 & 42.86 & 73.21 & 76.62 & 84.67 & 114.44 & 115.00 & 522.43 \\
\hline $\begin{array}{l}\text { NPK } \\
\text { briquette }\end{array}$ & 36.00 & 55.87 & 57.93 & 76.55 & 94.97 & 136.50 & 138.77 & 596.59 \\
\hline NP briquette & 30.76 & 53.50 & 55.73 & 75.50 & 94.33 & 135.10 & 137.33 & 582.26 \\
\hline UB & 23.33 & 52.89 & 53.92 & 72.83 & 88.33 & 129.83 & 120.77 & 541.91 \\
\hline CDU & 13.47 & 42.12 & 55.19 & 62.17 & 81.40 & 106.22 & 104.53 & 465.09 \\
\hline Control & 8.30 & 14.52 & 20.59 & 18.60 & 17.34 & 16.87 & 16.67 & 112.89 \\
\hline
\end{tabular}


Table.5 Nitrogen release potential of Entisol due to effect of nitrogenous fertilizers

\begin{tabular}{|l|c|c|c|c|c|c|c|c|}
\hline \multirow{2}{*}{$\begin{array}{c}\text { Nitrogenous } \\
\text { fertilizers }\end{array}$} & \multicolumn{7}{|c|}{ Incubation periods (Days) } & $\begin{array}{c}\text { Cumulative } \\
\text { total }\end{array}$ \\
\cline { 2 - 10 } NCU & 29.43 & 25.88 & 23.72 & 10.85 & 6.84 & 6.69 & 5.88 & 109.29 \\
\hline DAP & 78.43 & 26.47 & 21.55 & 9.05 & 7.37 & 5.96 & 5.27 & 154.10 \\
\hline $\begin{array}{l}\text { NPK } \\
\text { briquette }\end{array}$ & 31.64 & 23.93 & 15.03 & 15.03 & 9.60 & 8.72 & 7.83 & 111.79 \\
\hline NP briquette & 32.00 & 27.08 & 15.58 & 13.74 & 8.23 & 7.60 & 7.60 & 111.83 \\
\hline UB & 31.13 & 26.99 & 18.82 & 12.50 & 7.23 & 6.64 & 5.94 & 109.25 \\
\hline CDU & 32.80 & 22.00 & 19.65 & 11.66 & 6.13 & 5.83 & 6.02 & 104.10 \\
\hline Control & 14.77 & 13.39 & 10.31 & 8.29 & 6.30 & 6.55 & 3.98 & 63.58 \\
\hline
\end{tabular}

Table.6 Effect of nitrogenous fertilizers on cumulative $\mathrm{NH}_{4}{ }^{+}-\mathrm{N}$ content of Vertisol $\left(\mathrm{mg} \mathrm{kg}^{-1}\right)$

\begin{tabular}{|c|c|c|c|c|c|c|}
\hline \multirow[t]{2}{*}{$\begin{array}{l}\text { Nitrogenous } \\
\text { fertilizers }\end{array}$} & \multicolumn{2}{|c|}{ Mineral $\mathbf{N}$ at 0 days } & \multicolumn{2}{|c|}{$\begin{array}{c}\text { Mineral N after } 90 \\
\text { days }\end{array}$} & \multirow{2}{*}{$\begin{array}{c}\mathbf{N} \\
\text { Mineralization } \\
\text { Potential } \\
\text { mg kg }^{-1} \\
\mathrm{e}=(\mathbf{c}-\mathbf{a})+(\mathbf{d}-\mathbf{b})\end{array}$} & \multirow{2}{*}{$\begin{array}{c}\mathbf{N} \\
\text { Mineralization } \\
\text { Potential } \\
\text { mg kg }^{-1} \text { day }^{-1} \\
\mathbf{f = e} / \mathbf{9 0}\end{array}$} \\
\hline & $\begin{array}{c}\mathrm{NH}_{4}{ }^{+}-\mathrm{N} \\
\left(\mathrm{mg} \mathrm{kg}^{-1}\right) \\
\text { (a) }\end{array}$ & $\begin{array}{l}\mathrm{NO}_{3}^{-}-\mathrm{N} \\
\left(\mathrm{mg} \mathrm{kg}^{-1}\right) \\
\text { (b) }\end{array}$ & $\begin{array}{c}\mathrm{NH}_{4}^{+}-\mathrm{N} \\
\left(\mathrm{mg} \mathrm{kg}^{-1}\right) \\
\text { (c) }\end{array}$ & $\begin{array}{c}\mathrm{NO}_{3}{ }^{-}-\mathrm{N} \\
\left(\mathrm{mg} \mathrm{kg}^{-1}\right) \\
\text { (d) }\end{array}$ & & \\
\hline NCU & 30.80 & 14.57 & 114.60 & 575.01 & 644.24 & 7.16 \\
\hline DAP & 75.00 & 15.63 & 153.03 & 522.43 & 584.82 & 6.50 \\
\hline $\begin{array}{l}\text { NPK } \\
\text { briquette }\end{array}$ & 31.03 & 36.00 & 98.97 & 596.59 & 628.53 & 6.98 \\
\hline NP briquette & 33.83 & 30.76 & 106.76 & 582.26 & 624.43 & 6.94 \\
\hline UB & 29.13 & 23.33 & 95.16 & 541.91 & 584.60 & 6.50 \\
\hline CDU & 44.87 & 13.47 & 121.04 & 465.09 & 527.79 & 5.86 \\
\hline Control & 14.97 & 8.30 & 67.22 & 112.89 & 156.84 & 1.74 \\
\hline
\end{tabular}

Table.7 Effect of nitrogenous fertilizers on cumulative $\mathrm{NO}_{3}{ }^{-} \mathrm{N}$ content of Vertisol $\left(\mathrm{mg} \mathrm{kg}^{-1}\right)$

\begin{tabular}{|c|c|c|c|c|c|c|c|c|}
\hline \multirow{2}{*}{$\begin{array}{c}\text { Nitrogenous } \\
\text { fertilizers }(F)\end{array}$} & \multicolumn{7}{|c|}{ Incubation periods (Days) } & \multirow{2}{*}{$\begin{array}{c}\text { Cumulative } \\
\text { total }\end{array}$} \\
\hline & 0 & 15 & 30 & 45 & 60 & 75 & 90 & \\
\hline NCU & 12.55 & 35.38 & 77.16 & 78.04 & 87.04 & 121.68 & 122.88 & 534.74 \\
\hline DAP & 15.40 & 70.83 & 78.48 & 82.10 & 94.31 & 117.92 & 116.90 & 575.94 \\
\hline $\begin{array}{l}\text { NPK } \\
\text { briquette }\end{array}$ & 37.67 & 62.25 & 81.63 & 92.89 & 95.55 & 137.01 & 136.83 & 643.83 \\
\hline NP briquette & 36.96 & 61.30 & 80.03 & 92.33 & 94.47 & 136.83 & 136.57 & 638.49 \\
\hline UB & 25.03 & 58.56 & 79.25 & 81.14 & 91.88 & 135.73 & 132.83 & 604.43 \\
\hline CDU & 12.13 & 35.92 & 61.41 & 61.67 & 74.64 & 96.73 & 97.73 & 440.24 \\
\hline Control & 7.39 & 13.07 & 17.88 & 18.00 & 17.62 & 15.10 & 15.01 & 104.07 \\
\hline
\end{tabular}


Table.8 Nitrogen release potential of Vertisol due to effect of nitrogenous fertilizers

\begin{tabular}{|c|c|c|c|c|c|c|}
\hline \multirow[t]{2}{*}{$\begin{array}{l}\text { Nitrogenous } \\
\text { fertilizers }\end{array}$} & \multicolumn{2}{|c|}{ Mineral $\mathbf{N}$ at 0 days } & \multicolumn{2}{|c|}{$\begin{array}{c}\text { Mineral N after } 90 \\
\text { days }\end{array}$} & \multirow{2}{*}{$\begin{array}{c}\mathbf{N} \\
\text { Mineralization } \\
\text { Potential } \\
\text { mg kg }^{-1} \\
\mathrm{e}=(\mathbf{c}-\mathbf{a})+(\mathbf{d}-\mathbf{b})\end{array}$} & \multirow{2}{*}{$\begin{array}{c}\mathbf{N} \\
\text { Mineralization } \\
\text { Potential } \\
\text { mg kg }^{-1} \text { day }^{-1} \\
\mathrm{f}=\mathrm{e} / 90\end{array}$} \\
\hline & $\begin{array}{c}\mathrm{NH}_{4}^{+}-\mathrm{N} \\
\left(\mathrm{mg} \mathrm{kg}^{-1}\right) \\
\text { (a) }\end{array}$ & $\begin{array}{l}\mathrm{NO}_{3}^{-}-\mathrm{N} \\
\left(\mathrm{mg} \mathrm{kg}^{-1}\right) \\
\text { (b) }\end{array}$ & $\begin{array}{c}\mathrm{NH}_{4}^{+}-\mathrm{N} \\
\left(\mathrm{mg} \mathrm{kg}^{-1}\right) \\
\text { (c) }\end{array}$ & $\begin{array}{c}\mathrm{NO}_{3}^{-}-\mathrm{N} \\
\left(\mathrm{mg} \mathrm{kg}^{-1}\right) \\
\text { (d) }\end{array}$ & & \\
\hline NCU & 29.43 & 12.55 & 109.29 & 534.74 & 602.04 & 6.69 \\
\hline DAP & 78.43 & 15.40 & 154.10 & 575.94 & 636.21 & 7.07 \\
\hline NPK briquette & 31.64 & 37.67 & 111.79 & 643.83 & 686.31 & 7.63 \\
\hline NP briquette & 32.00 & 36.96 & 111.83 & 638.49 & 681.36 & 7.57 \\
\hline UB & 31.13 & 25.03 & 109.25 & 604.43 & 657.52 & 7.31 \\
\hline CDU & 32.80 & 12.13 & 104.10 & 440.24 & 499.40 & 5.55 \\
\hline Control & 14.77 & 7.39 & 63.58 & 104.07 & 145.50 & 1.62 \\
\hline
\end{tabular}

In conclusion in case of Entisol the highest $\mathrm{N}$ release potential is observed through application of neem coated urea and followed by NPK briquette whereas in case of Vertisol the highest $\mathrm{N}$ release potential is observed through application of NPK briquette and followed by NP briquette. The application of all the sources of nitrogenous fertilizers increased the $\mathrm{N}$ release potential by $336.78 \%$ to $411.49 \%$ over the control in Entisol and by $342.59 \%$ to $470.98 \%$ over the control in Vertisol.

\section{References}

Cabrera, M. L., Kissel, D. E. and Vigil, M. F. 2005. Nitrogen mineralization from organic residues. Journal of Environmental Quality, 34: 75-79.

Daftardar, S.V. and Savant, N.K. (1995) Evaluation of environmental friendly fertilizer management for rainfed Low land rice on tribal farmer's field in India. Paper presented at IRRC, 13-17 Feb., 1995. Los Banos, Laguna, Philippines.

Durgude A.G., Y.J. Patil, A.V. Bulbule and V.S. Patil (2008) Effect of fertilizer management through urea - DAP briquettes on low land rice. Asian Journal of Soil Science, 3: 1-3.
Keeney, D. R. and Nelson, D.W. (1982). Nitrogen-Inorganic forms. In "Methods of Soil Analysis, Part Chemical and Microbiological Properties", Page A. L. (Ed), II edition, American Society of Agronomy, Inc. and Soil Science Society of America. Inc. Madison, Wisconsin, USA, 643-693.

More N. B. 1999. Use of NPK briquette in Sugarcane. Ph.D. thesis submitted to M.P.K.V., Rahuri.

More N.B. and B.N. Shinde (2001) Effect of NPK briquette on Availability and Uptake of Nutrients by Sugarcane. Journal of Maharashtra Agricultural Universities, 27: 121-123.

Singh K. K. (2012) Use of NPK briquette for wheat, M.sc. (Agri) thesis submitted to M.P.K.V., Rahuri.

SinghSubhash (2017) Kinetics of nitrogen mineralization in Inceptisol by the use of organic manures, M.Sc. agri thesis submitted to MPKV, Rahuri.

Suganya S., K. Appavu and A. Vadivel (2009) Mineralization pattern of neem coated urea products in different soils. Research Paper. International Journal of Agricultural Science, 5: 175-179.

\section{How to cite this article:}

More, N.B., S.S. Sadafule, V.R. Gaikwad, B.D. Tamboli and Patil, G.D. 2019. Study of Nitrogen Release Potential of Entisol and Vertisol as Influenced by Various Sources of Nitrogenous Fertilizers. Int.J.Curr.Microbiol.App.Sci. 8(01): 2950-2955. doi: https://doi.org/10.20546/ijcmas.2019.801.313 